PROCEEDINGS OF THE

AMERICAN MATHEMATICAL SOCIETY

Volume 126, Number 4, April 1998, Pages 999-1004

S 0002-9939(98)04213-0

\title{
COMMON ZEROS OF THETA FUNCTIONS AND CENTRAL HECKE L-VALUES OF CM NUMBER FIELDS OF DEGREE 4
}

\author{
TONGHAI YANG
}

(Communicated by William W. Adams)

\begin{abstract}
In this note, we apply the method of Rodriguez Villegas and Yang (1996) to construct a family of infinite many theta series over the HilbertBlumenthal modular surfaces with a common zero. We also relate the nonvanishing of the central L-values of certain Hecke characters of non-biquadratic $\mathrm{CM}$ number fields of degree 4 to the nonvanishing of theta functions at $\mathrm{CM}$ points in the Hilbert-Blumenthal modular surfaces.
\end{abstract}

\section{INTRODUCTION}

It is well-known that the theta function $\theta(z)=\sum e\left(x^{2} z\right)$ has no zeros in the upper half plane, where $e(z)=e^{2 \pi i z}$. It was proved in [RVY] that, for integers $d \equiv 1 \bmod 4$ and $k \geq 0$, whether the theta functions

$$
\theta_{d, k}(z)=(\operatorname{Im}(2 z))^{-\frac{k}{2}} \sum_{(x, d)=1}\left(\frac{d}{x}\right) H_{k}(x \sqrt{\operatorname{Im}(2 z)}) e\left(x^{2} z\right)
$$

vanish at certain Heegner points is related to the vanishing of central Hecke L-values ([RVY, Theorem 0.2]). Here $H_{k}$ is a suitably normalized Hermite polynomial. Over a real quadratic field $F=\mathbb{Q}(\sqrt{p})$, one can also define a theta function $\theta_{d, k}$ on the Hilbert upper half plane $\mathcal{H}^{2}$ in the same manner. In fact, one can replace $(\stackrel{d}{)})$ by any quadratic Hecke character of $F$. (See (1) and (10) for the exact meaning). They are nonholomorphic Hilbert modular forms of weight $k+\frac{1}{2}$ for some congruence group $\Gamma_{d}$ over $F$. We remark that $\Gamma_{d}$ is independent of $k$. The first result involves the zero locus of these theta functions. We prove that those theta functions sometimes have a common zero when $k$ varies.

Theorem 0. Let $p=5,13$, or 61 . Let $d \equiv 1 \bmod 4$ be a rational integer such that every prime factor of $d$ is split in either $\mathbb{Q}(\sqrt{-7})$ or $\mathbb{Q}(\sqrt{-7 p})$.

(a) If $d<0$, then $\left\{\theta_{d, k}: k \geq 0\right.$, even $\}$ has a common zero in $\mathbb{Q}(\sqrt{p}, \sqrt{-7}) \cap \mathcal{H}^{2}$.

(b) If $d>0$, then $\left\{\theta_{d, k}: k \geq 1\right.$, odd $\}$ has a common zero in $\mathbb{Q}(\sqrt{p}, \sqrt{-7}) \cap \mathcal{H}^{2}$.

Here we embed $\mathbb{Q}(\sqrt{p}, \sqrt{-7})$ into $\mathbb{C}^{2}$ via $z \mapsto\left(\sigma_{1}(z), \sigma_{2}(z)\right)$, where $\sigma_{j}(\sqrt{p})=$ $(-1)^{j-1} \sqrt{p}$ and $\sigma_{j}(\sqrt{-7})=i \sqrt{7}$ for $j=1,2$.

Received by the editors September 27, 1996.

1991 Mathematics Subject Classification. Primary 11F27, 11F67, 11M06.

Key words and phrases. Theta function, central Hecke L-value, ideal class number.

The author was partially supported by NSF grant DMS-9304580.

(C)1998 American Mathematical Society 
The theorem is also true if we replace $p$ by 17 and -7 by -11 . The proof of this theorem and its generalization will be given in section 2 . In this note, we will also relate the central Hecke L-value to the values of theta functions at CM points and relate the vanishing of the Hecke L-value to the CM zeros of the theta functions (Theorem 2 and 8 ).

\section{Notation AND PRELIMINARIES}

Assume $F=\mathbb{Q}(\sqrt{p})$ is a real quadratic number field with $p \equiv 1 \bmod 4$. We view $F$ as a subfield of $\mathbb{R}$ with $\sqrt{p}>0$, so the other embedding of $F$ into $\mathbb{R}$ is given by $x=a+b \sqrt{p} \mapsto x^{\prime}=a-b \sqrt{p}, a, b \in \mathbb{Q}$. We embed $F$ into $\mathbb{C}^{2}$ via $x \mapsto\left(x, x^{\prime}\right)$. Let $\mathcal{H}^{2}=\left\{z=\left(z_{1}, z_{2}\right) \in \mathbb{C}^{2}: \operatorname{Im} z_{i}>0\right\}$ be the Hilbert upper plane. Given a function $\phi$ on $\mathbb{C}$, we define a function on $\mathbb{C}^{2}$, still denoted by $\phi$, via $\phi(z)=\phi\left(z_{1}\right) \phi\left(z_{2}\right)$. Let $u$ be a fixed unit of $F$ with $u>0$ and $u^{\prime}<0$. Let $\mu$ be a quadratic Hecke character of $F$ with principal conductor $f \mathcal{O}_{F}$; then $\mu$ induces a character $\mu:\left(\mathcal{O}_{F} / f\right)^{*} \longrightarrow \mathbb{C}^{1}$. We assume $\mu(-1)=1$. Multiplying by $u$ if necessary, we may assume that $f$ is totally positive. For a rational integer $k \geq 0$, we define a theta function $\theta_{\mu, k}$ on the Hilbert upper plane $\mathcal{H}^{2}$ via

$$
\theta_{\mu, k}(z)=(\operatorname{Im}(2 z))^{-\frac{k}{2}} \sum_{\substack{x \in \mathcal{O}_{F} \\(x, f)=1}} \mu(x) H_{k}(x \sqrt{\operatorname{Im}(2 z)}) e\left(x^{2} z\right) .
$$

Here $e(z)=e^{2 \pi i z}$, and $H_{k}$ is the $k$ th Hermite polynomial normalized via [RVY, $(0.13)]$. The theta function $\theta_{\mu, k}$ is a nonholomorphic Hilbert modular form of weight $k+\frac{1}{2}$ for some congruence group $\Gamma_{d}$ over $F$ and is holomorphic when $k=0$ or 1 . We notice that $\Gamma_{d}$ is independent of $k$.

Let $D$ be a totally positive integer in $\mathcal{O}_{F}$, and let $E=F(\sqrt{-D})$ be a totally imaginary extension of $F$. We assume throughout this paper

(2) $D \mathcal{O}_{F}$ is prime, and $E / F$ is ramified only at $D \mathcal{O}_{F}$,

(3) every prime of $F$ dividing $2 f \mathcal{O}_{F}$ is split in $E / F$, and

(4) $E$ is generic in the sense of Rohrlich ([Roh, page 519]), that is, $\mathcal{O}_{E}^{*}=\mathcal{O}_{F}^{*}$ and $\mathrm{CL}(F) \hookrightarrow \mathrm{CL}(E)$ naturally.

Let $\Phi=\left\{\sigma_{1}, \sigma_{2}\right\}$ be the CM type of $E$ given by

$$
\sigma_{1}(\sqrt{p})=\sqrt{p}, \quad \sigma_{1}(\sqrt{-D})=i \sqrt{D}
$$

and

$$
\sigma_{2}(\sqrt{p})=-\sqrt{p}, \quad \sigma_{2}(\sqrt{-D})=i \sqrt{D^{\prime}} .
$$

Here $\sqrt{a}$ stands for the positive square root of $a$ for $a>0$. We embed $E$ into $\mathbb{C}^{2}$ via $z \mapsto\left(\sigma_{1}(z), \sigma_{2}(z)\right)$.

Let $\tilde{\mu}=\mu \circ N_{E / F}$; then $\left.\tilde{\mu}\right|_{F_{\mathbb{A}}^{*}}=1$ and there is a character $\eta$ of $E^{1} \backslash E_{\mathbb{A}}^{1}$ such that $\tilde{\mu}=\tilde{\eta}$, where $\tilde{\eta}(z)=\eta(z / \bar{z})$. Let $\chi_{\text {can }}$ be a canonical Hecke character of $E$ of infinite type $\Phi$ in the sense of Rohrlich ([Roh, page 518]). Then the character $\chi_{\text {can }} \tilde{\mu}$ is closely realted to CM abelian varieties ([Sh]) and has some simple properties ([RVY, Lemma 2.0]). Let $\chi=\chi_{\text {can }}||_{\mathbb{A}}^{\frac{1}{2}}$, then $\left.\chi\right|_{F_{\mathbb{A}}^{*}}=\epsilon$ is the quadratic Hecke character of $F$ associated to $E / F$. Denote $\eta_{k}=\left.\eta \chi^{k}\right|_{E_{\AA}^{1}}$, so $\tilde{\eta}_{k}=\tilde{\eta} \chi^{2 k}$. Let $\psi=\prod \psi_{v}$ be the 'canonical' additive character of $F_{\mathbb{A}} / F$ given by

$$
\psi_{v}(x)= \begin{cases}e^{2 \pi i x} & \text { if } v \text { is real } \\ e^{-2 \pi i \lambda(x)} & \text { if } v \text { is finite }\end{cases}
$$


where $\lambda: F_{v} \stackrel{\operatorname{tr}}{\longrightarrow} \mathbb{Q}_{p} \longrightarrow \mathbb{Q}_{p} / \mathbb{Z}_{p} \hookrightarrow \mathbb{Q} / \mathbb{Z}$. Let $\psi_{E}=\psi \circ \operatorname{tr}_{E / F}$.

Lemma 1. Let $\alpha=\frac{4 u}{D \sqrt{p}}$ and $\delta=\sqrt{-D}$. Then for any rational integer $k \geq 0$, the datum $\left(\chi, \eta_{k}, \alpha, \delta, \psi, \Phi\right)$ satisfies (0.4), (0.6), and (0.9) in [RVY].

Proof. It is easy to see that

$$
\epsilon_{v}(\alpha)= \begin{cases}\epsilon_{\sqrt{p}}(\sqrt{p}) & \text { if } v=\sqrt{p} \mathcal{O}_{F} \text { or } D \mathcal{O}_{F}, \\ 1 & \text { otherwise. }\end{cases}
$$

Here we have abused the notation by using $\sqrt{p}$ for the place of $F$ corresponding to the prime ideal $\sqrt{p} \mathcal{O}_{F}$. Since $n\left(\psi_{v}\right)=-1$ when $v=\sqrt{p} \mathcal{O}_{F}$ and $n\left(\psi_{v}\right)=0$ otherwise, one has, by [RVY, Lemma 2.3],

$$
\epsilon\left(\frac{1}{2},\left(\chi \tilde{\eta}_{k}\right)_{v}, \frac{1}{2} \psi_{E_{v}}\right)\left(\chi \tilde{\eta}_{k}\right)_{v}(\delta)= \begin{cases}? & \text { if } v=D \mathcal{O}_{F} \\ \epsilon_{\sqrt{p}}(\sqrt{p}) & \text { if } v=\sqrt{\mathcal{O}}_{F}, \\ 1 & \text { otherwise. }\end{cases}
$$

But the global root number $\epsilon\left(\frac{1}{2}, \chi \tilde{\eta}_{k}\right)=\mu(-1)(-1)^{2 k}=1$ by [RVY, Lemma 2.3]. So $?=\epsilon_{\sqrt{p}}(\sqrt{p})$. Therefore

$$
\epsilon\left(\frac{1}{2},\left(\chi \tilde{\eta}_{k}\right)_{v}, \frac{1}{2} \psi_{E_{v}}\right)\left(\chi \tilde{\eta}_{k}\right)_{v}(\delta)=\epsilon_{v}(\alpha)
$$

for every place $v$ of $F$. This verifies condition (0.6) in [RVY]. The verification of [RVY, (0.4) and (0.9)] is trivial.

Now applying [RVY, Theorem 2.7], one has

Theorem 2. Notation as above. Assume that $F$ has ideal class number 1 . Fix a square root $r$ of $-D \bmod 16 f^{2}$. For every ideal class $C \in \mathrm{CL}(E)$, choose a primitive ideal $\mathfrak{A} \in C^{-1}$ relatively prime to $2 f$, and write

$$
\mathfrak{A}^{2}=\left[a^{2}, \frac{b+\sqrt{-D}}{2}\right]
$$

with a totally positive, and

$$
b \equiv r \bmod 8 f^{2} .
$$

Then

$$
L\left(k+1,\left(\chi_{\operatorname{can} \tilde{\mu}}\right)^{2 k+1}\right)=\kappa\left|\sum_{C \in \operatorname{CL}(E)} \frac{\mu(a)}{\left(\chi_{\operatorname{can} \tilde{\mu})^{2 k+1}(\overline{\mathfrak{A}})}\right.} \theta_{\mu, k}\left(\tau_{\mathfrak{A}}\right)\right|^{2} .
$$

Here

$$
\kappa=\frac{\pi^{2 k+2}}{(k !)^{2} \sqrt{N_{F / \mathbb{Q}} D}}\left(N_{F / \mathbb{Q}} \frac{D}{p f^{4}}\right)^{\frac{2 k+1}{4}},
$$

and

$$
\tau_{\mathfrak{A}}=\frac{u(b+\sqrt{-D})}{8 \sqrt{p} f^{2} a^{2}}
$$


For a CM type $\Phi$ of $E$, we write $k \Phi$ for $\sum_{\sigma \in \Phi} k \sigma$. According to Rohrlich ([Roh2, page 700$]$ ), there is a group homomorphism

$$
h_{k \Phi}: \quad \mathrm{CL}(E) \longrightarrow \mathrm{CL}\left(E^{k \Phi}\right) .
$$

Here $E^{k \Phi}$ is the number field generated by $z^{k \Phi}=\prod \sigma(z)^{k}, z \in E^{*}$. Applying [RVY, Theorem 2.8], one has

Corollary 3. Notation and assumption as in Theorem 2. Assume further that $h_{(2 k+1) \Phi}$ is injective. Then the following statements are equivalent.

(a) The central L-value $L\left(k+1,\left(\chi_{\mathrm{can}} \tilde{\mu}\right)^{2 k+1}\right)=0$.

(b) The global theta lifting $\theta_{\alpha, \chi}\left(\eta_{k}\right)=0$.

(c) For every ideal class $C \in \mathrm{CL}(E), I_{C}\left(\eta_{k}\right)=0$.

(d) For every ideal class $C \in \mathrm{CL}(E), \tau_{\mathfrak{A}}$ is a root of the theta function $\theta_{\mu, k}$.

In general, one has

Proposition 4. Notation as in Lemma 1. If $\mathrm{CL}(F) \supset \operatorname{ker} h_{(2 k+1) \Phi}$, and the central $L$-value $L\left(k+1,\left(\chi_{\mathrm{can}} \tilde{\mu}\right)^{2 k+1}\right)=0$, then $\tau=\frac{u(b+\sqrt{-D})}{8 \sqrt{p} f^{2}} \in E$ is a root of the Hilbert modular form $\theta_{\mu, k}$. Here $b \in \mathcal{O}_{F}$ satisfies $b^{2} \equiv-D \bmod 16 f^{2}$.

Proof. Applying the argument in the proof of [RVY, Theorem 2.8] to [RVY, Theorem 2.6], one has that $2 u(b+\sqrt{-D})$ is a root of $\theta_{\mu, k, \mathcal{O}_{F}}$, where $\theta_{\mu, k, \mathcal{O}_{F}}$ is given by [RVY, (2.3)]. Simple manipulation gives

$$
\theta_{\mu, k, \mathcal{O}_{F}}(z)=\gamma^{\prime} N_{F / \mathbb{Q}}(4 f)^{-k} \theta_{\mu, k}\left(\frac{z}{16 f^{2}}\right)
$$

where $\gamma^{\prime}=\prod_{v \mid f} \frac{G\left(\frac{1}{4 f} \psi_{v}, \mu_{v}\right)}{\sqrt{q_{v}}} \in \mathbb{C}^{1}$. So $\tau$ is a root of $\theta_{\mu, k}$.

\section{Common zeros of theta functions}

Recall that $F=\mathbb{Q}(\sqrt{p})$, with $p \equiv 1 \bmod 4$ being a prime. Let $d \equiv 1 \bmod 4$ be a rational integer relatively prime to $p$, and let $\epsilon^{d}$ be the Hecke character of $\mathbb{Q}$ associated to the Dirichlet character $(\underline{d})$, and let $\mu=\epsilon^{d} \circ N_{F / \mathbb{Q}}$. Denote $\theta_{d, k}=\theta_{\mu, k}$, i.e.,

$$
\theta_{d, k}(z)=(\operatorname{Im}(2 z))^{-\frac{k}{2}} \sum_{x \in \mathcal{O}_{F}}\left(\frac{d}{x x^{\prime}}\right) H_{k}(x \sqrt{\operatorname{Im}(2 z)}) e\left(x^{2} z\right) .
$$

For a rational prime number $q \equiv 3 \bmod 4$, let $E=\mathbb{Q}(\sqrt{p}, \sqrt{-q})$ and $F_{2}=\mathbb{Q}(\sqrt{-q})$. For an integer $k \geq 0$, let $N^{k}: \mathrm{CL}(E) \longrightarrow \mathrm{CL}\left(F_{2}\right)$ be the map induced by $\mathfrak{A} \mapsto$ $\left(N_{E / F_{2}}(\mathfrak{A})\right)^{2 k+1}$.

Proposition 5. Let $q \equiv 3 \bmod 4$ be a rational prime number such that $(q / p)=-1$ and every prime factor of $2 d$ is split in either $\mathbb{Q}(\sqrt{-q})$ or $\mathbb{Q}(\sqrt{-p q})$. Assume further that $q>3$ and $\mathrm{CL}(F) \supset \operatorname{ker} N^{k}$. Let $b \in \mathcal{O}_{F}$ be an algebraic integer in $F$ such that $b^{2} \equiv-q \bmod 16 d^{2}$.

(a) If $d<0$ and $k$ is even, then $\tau=\frac{u(b+\sqrt{-q})}{8 \sqrt{p} d^{2}}$ is a root of $\theta_{d, k}$.

(b) If $d>0$ and $k$ is odd, then $\tau=\frac{u(b+\sqrt{-q})}{8 \sqrt{p} d^{2}}$ is a root of $\theta_{d, k}$.

Proof. Since $p$ is a prime, $\mathrm{CL}(F)$ is an odd group, and so the canonical map $\mathrm{CL}(F) \longrightarrow \mathrm{CL}(E)$ is injective. Since $q>3, \mathcal{O}_{E}^{*}=\mathcal{O}_{F}^{*}$. So $E$ is generic. Let $\chi_{\mathrm{can}, 2}$ be a canonical Hecke character of $F_{2}$, and let $\chi_{\mathrm{can}}=\chi_{\mathrm{can}, 2} \circ N_{E / F_{2}}$; then 
$\chi_{\text {can }}$ is a canonical Hecke character of $E$ of infinite type $\Phi=\left\{\sigma_{1}, \sigma_{2}\right\}$. Moreover, one has

$$
L\left(s, \chi_{\mathrm{can}}^{2 k+1}\left(\frac{d}{-}\right) \circ N_{E / \mathbb{Q}}\right)=L\left(s, \chi_{\mathrm{can}, 2}^{2 k+1}\left(\frac{d}{-}\right) \circ N_{F_{2} / \mathbb{Q}}\right) L\left(s,\left(\chi_{\mathrm{can}, 2}^{2 k+1}\left(\frac{d}{-}\right) \circ N_{F_{2} / \mathbb{Q}}\right)^{\sigma_{2}}\right) .
$$

Under either condition $(a)$ or $(b)$, the global root number of $\chi_{\mathrm{can}, 2}^{2 k+1}(\underline{d}) \circ N_{F_{2} / \mathbb{Q}}$ is -1 , and so the central L-value $L\left(k+1, \chi_{\mathrm{can}, 2}^{2 k+1}(\underline{d}) \circ N_{F_{2} / \mathbb{Q}}\right)=0$. This implies $L\left(k+1, \chi_{\text {can }}^{2 k+1}(\underline{d}) \circ N_{E / \mathbb{Q}}\right)=0$. Notice that $h_{(2 k+1) \Phi}=N^{k}$ in this case. Now applying Proposition 4 , one proves the proposition.

Corollary 6. Notation as in Proposition 5. Assume $q>3$ and $E=\mathbb{Q}(\sqrt{p}, \sqrt{-q})$ has ideal class number 1 .

(a) If $d<0$, then $\tau=\frac{u(b+\sqrt{-q})}{8 \sqrt{p} d^{2}}$ is a common root of $\theta_{d, k}$ for all even integers $k \geq 0$.

(b) If $d>0$, then $\tau=\frac{u(b+\sqrt{-q})}{8 \sqrt{p} d^{2}}$ is a common root of $\theta_{d, k}$ for all odd integers $k>0$.

Proof of Theorem 0. According to [Yam, Table], the biquadratic CM fields satisfying all the conditions in Corollary 6 are $\mathbb{Q}(\sqrt{p}, \sqrt{-7})$ with $p=5,13,61$ and $\mathbb{Q}(\sqrt{17}, \sqrt{-11})$. This proves Theorem 0 .

\section{Nonbiquadratic CM Number fields of Degree 4}

Let the notation and assumptions be as in section 1. We further assume that $D$ is not a rational integer. Write $D=x+y \sqrt{p}>0$ with $x, y \in \frac{1}{2} \mathbb{Z}, x \equiv y \bmod \mathbb{Z}$, and $y \neq 0$. Then $D^{\prime}=x-y \sqrt{p}>0$ and $q=D D^{\prime}$ is a positive rational prime number. When $q=p, E$ is cyclic over $\mathbb{Q}$ and is a subfield of $\mathbb{Q}\left(\zeta_{p}\right)$. In this case, $E^{\Phi}=E$. When $q \neq p$, let $K=E\left(\sqrt{-D^{\prime}}\right)$, and identify it as a subfield of $\mathbb{C}$ via

$$
\sigma_{1}: \quad K \longrightarrow \mathbb{C} ; \quad \sqrt{p} \mapsto \sqrt{p}, \quad \sqrt{-D} \mapsto i \sqrt{D}, \quad \sqrt{-D^{\prime}} \mapsto i \sqrt{D^{\prime}} .
$$

Let

$$
\delta=\sqrt{-D}=i \sqrt{D}, \quad \delta^{\prime}=\sqrt{-D^{\prime}}=i \sqrt{D^{\prime}}
$$

and

$$
\tilde{\delta}=\delta+\delta^{\prime}, \quad \tilde{\delta}^{\prime}=\delta-\delta^{\prime} .
$$

Then $\delta \delta^{\prime}=-q$ and $\tilde{\delta} \tilde{\delta}^{\prime}=-2 y p$. Furthermore, let $E^{\prime}=F\left(\delta^{\prime}\right), \tilde{F}=\mathbb{Q}(\sqrt{q})$, $\tilde{E}=\tilde{F}(\tilde{\delta})$, and $\tilde{E}^{\prime}=\tilde{F}\left(\tilde{\delta}^{\prime}\right)$. Then $E^{\prime}, \tilde{E}$, and $\tilde{E}^{\prime}$ are all subfields of $K$, and $K$ is a dihedral extension of $\mathbb{Q}$ of degree 8. Write $\operatorname{Gal}(K / \mathbb{Q})=\langle\sigma, \tau\rangle$ with

$$
\sigma: \delta \mapsto \delta^{\prime}, \quad \delta^{\prime} \mapsto-\delta, \quad \tilde{\delta} \mapsto-\tilde{\delta}^{\prime}, \quad \tilde{\delta}^{\prime} \mapsto \tilde{\delta}, \quad \sqrt{p} \mapsto-\sqrt{p}, \quad \sqrt{q} \mapsto-\sqrt{q},
$$

and

$$
\tau: \delta \mapsto \delta, \quad \delta^{\prime} \mapsto-\delta^{\prime}, \quad \tilde{\delta} \mapsto \tilde{\delta}^{\prime}, \quad \tilde{\delta}^{\prime} \mapsto \tilde{\delta}, \quad \sqrt{p} \mapsto \sqrt{p}, \quad \sqrt{q} \mapsto-\sqrt{q} .
$$

Notice that $\Phi=\{1, \sigma\}$. Let $\tilde{\Phi}=\{1, \tau\}$; then $\tilde{\Phi}$ is a CM type of $\tilde{E}$. One has $E^{\Phi}=\tilde{E}$ and $\tilde{E}^{\tilde{\Phi}}=E$. So for every integer $n>0$ there is a map

$$
h_{n \tilde{\Phi}} \circ h_{n \Phi}: \quad \mathrm{CL}(E) \longrightarrow \mathrm{CL}(\tilde{E}) \longrightarrow \mathrm{CL}(E) .
$$

Proposition 7. Assume that $F$ has ideal class number 1.

(a) When $q \neq p$, the map $h_{n \tilde{\Phi}} \circ h_{n \Phi}$ is the $(2 n)$-th power map.

(b) When $q=p, h_{n \Phi}^{2}$ is the (2n)-th power map. 
Proof. Given an ideal $\mathfrak{A}$ of $E$, we also use $\mathfrak{A}$ for its ideal class in $\operatorname{CL}(E)$. We onle prove the case $n=1$, as the others are the same. By definition ([Roh2, page $700]), h_{\Phi}(\mathfrak{A})=\left(\mathfrak{A} \sigma(\mathfrak{A}) \mathcal{O}_{K}\right) \cap \mathcal{O}_{\tilde{E}}$. Since $\operatorname{Gal}(K / \tilde{E})=\left\{1, \tau \sigma^{3}\right\}=\{1, \sigma \tau\}$, and $\tau \in \operatorname{Gal}(K / E)$, one has

$$
\begin{aligned}
h_{\Phi}(\mathfrak{A}) \mathcal{O}_{K} & =\left(\mathfrak{A} \sigma(\mathfrak{A}) \mathcal{O}_{K}\right) \cap\left(\tau \sigma^{3}(\mathfrak{A}) \tau(\mathfrak{A}) \mathcal{O}_{K}\right) \\
& =\left(\mathfrak{A} \sigma(\mathfrak{A}) \mathcal{O}_{K}\right) \cap\left(\sigma \tau(\mathfrak{A}) \mathfrak{A O}_{K}\right) \\
& =\mathfrak{A} \sigma(\mathfrak{A}) \mathcal{O}_{K} .
\end{aligned}
$$

So

$$
\begin{aligned}
h_{\tilde{\Phi}} \circ h_{\Phi}(\mathfrak{A}) & =\left(\mathfrak{A} \sigma(\mathfrak{A}) \mathcal{O}_{K}\right)^{\tilde{\Phi}} \cap E \\
& =\mathfrak{A} \sigma(\mathfrak{A}) \mathcal{O}_{K} \tau\left(\mathfrak{A} \sigma(\mathfrak{A}) \mathcal{O}_{K}\right) \cap E \\
& =\mathfrak{A}^{2} \sigma(\mathfrak{A}) \tau \sigma(\mathfrak{A}) \mathcal{O}_{K} \cap E \\
& =\mathfrak{A}^{2} \sigma(\mathfrak{A}) \sigma^{3}(\mathfrak{A}) \mathcal{O}_{K} \cap E \\
& =\mathfrak{A}^{2} N_{E^{\prime} / F}(\sigma(\mathfrak{A})) \\
& =\mathfrak{A}^{2}
\end{aligned}
$$

since $F$ has ideal class number 1 . This proves $(a)$. Claim $(b)$ can be proved similarly.

Now applying Corollary 3, we immediately get

Theorem 8. Assume that $F$ has ideal class number 1 , and that $E=F(\sqrt{-D})$ satisfies conditions (2)-(4). Assume further that $D$ is not a rational number and $\left(2(2 k+1), h_{E}\right)=1$, where $h_{E}$ is the ideal class number of $E$. Then the central L-value

$$
L\left(k+1,\left(\chi_{\left.\operatorname{can} \tilde{\mu})^{2 k+1}\right)=0}\right.\right.
$$

if and only if for every ideal class $C \in \mathrm{CL}(E)$ and a (and every) primitive ideal $\mathfrak{A} \in C^{-1}$ relatively prime to $2 f$, the CM point $\tau_{\mathfrak{A}}$ in Theorem 2 is a root of the Hilbert modular form $\theta_{\mu, k}$.

The author would like to thank the Institute for Advanced Study for its hospitality and financial support during the preparation of this note.

\section{REFERENCES}

[Roh] D. Rohrlich, Root numbers of Hecke L-functions of CM fields, Amer. J. Math. 104 (1982), 517-543. MR 83j:12011

[Roh2] _ Galois conjugacy of unramified twists of Hecke characters, Duke Math J. 47 (1980), 695-703. MR 82a:12009

[RVY] F. Rodriguez Villegas and T. H. Yang, Central values of Hecke L-functions of CM number fields, submitted to Duke Math. J., 1996.

[Sh] G. Shimura, Introduction to the arithmetic theory of automorphic functions, vol. 11, Publ. Math. Soc. Japan, 1971. MR 47:3318

[Yam] K. Yamamura, The determination of the imaginary abelian number fields with class number one, J. Number Theory 62 (1994), 899-921. MR 94g:11096

Department of Mathematics, University of Michigan, Ann Arbor, Michigan 48109

E-mail address: thyang@math.1sa.umich.edu 